\title{
Prompt particle emission in fission - news on systematics and predictions for fission induced by fast neutrons
}

\author{
Andreas Oberstedt ${ }^{1, a}$ and Stephan Oberstedt ${ }^{2}$ \\ ${ }^{1}$ Extreme Light Infrastructure - Nuclear Physics (ELI-NP) / Horia Hulubei National Institute for Physics and Nuclear Engineering \\ (IFIN-HH), 077125 Bucharest-Magurele, Romania \\ 2 European Commission, DG Joint Research Centre, Directorate G - Nuclear Safety and Security, Unit G.2 Standards for Nuclear \\ Safety, Security and Safeguards, 2440 Geel, Belgium
}

\begin{abstract}
As a consequence of recent experimental results, previously established systematics for prompt fission $\gamma$-ray spectra (PFGS) characteristics as function of both atomic and mass number of the compound system have been revised. Although based on purely empirical dependences, it allows estimating average gamma-ray multiplicity, mean and total photon energy in cases, where the target nuclei are either not available or not accessible experimentally. Based on this systematics, we show in this paper that PFGS characteristics may also be predicted for fission induced by fast neutrons. Our calculations were performed for the target nuclei ${ }^{238} \mathrm{U},{ }^{235} \mathrm{U}$ and ${ }^{239} \mathrm{Pu}$ in the neutron energy range from 0 to $20 \mathrm{MeV}$, and the results are compared to existing experimental and theoretical values.
\end{abstract}

\section{Introduction}

In recent years the measurement of prompt fission $\gamma$-ray spectra (PFGS) has gained renewed interest. After about forty years since the first comprehensive studies on this topic, the development of lanthanide halide scintillation detectors as well as new data acquisition and signal-processing techniques provided appropriate tools to determine PFGS characteristics, i.e. average total $\gamma$-ray energy released in prompt fission, mean energy per photon as well as average photon multiplicity, with unprecedented accuracy. These new experimental efforts were motivated by OECD/NEA requests for new values especially for gamma-ray multiplicities and mean photon energies, in particular for ${ }^{235} \mathrm{U}(\mathrm{n}, \mathrm{f})$ and ${ }^{239} \mathrm{Pu}(\mathrm{n}, \mathrm{f})$ [1]. Both target isotopes are considered the most important ones with respect to the modeling of innovative cores for fast Generation-IV reactors [2].

Based on the latest experimental results from the reactions ${ }^{235} \mathrm{U}\left(\mathrm{n}_{t}, \mathrm{f}\right)$ [3] and ${ }^{241} \mathrm{Pu}\left(\mathrm{n}_{t h}, \mathrm{f}\right)$ [4] as well as from the spontaneous fission of ${ }^{252} \mathrm{Cf}[5,6]$, we have recently presented a revised version of systematics for PFGS characteristics as function of both atomic and mass number of the compound systems undergoing fission [7,8], originally established already in 2001 [9]. In the meantime, new results have been obtained from the spontaneous fission of ${ }^{240} \mathrm{Pu}$ and ${ }^{242} \mathrm{Pu}$ [10], which now have been included in order to update the systematics. Below we will show that this systematics allows estimating PFGS characteristics in cases, where target nuclei are not available or accessible experimentally. Although this was applied before to thermal-neutron induced and spontaneous fission only, we will demonstrate in this work how PFGS characteristics may even be predicted for fission induced by fast neutrons. Examples are given for ${ }^{238} \mathrm{U}(\mathrm{n}, \mathrm{f}),{ }^{235} \mathrm{U}(\mathrm{n}, \mathrm{f})$ and ${ }^{239} \mathrm{Pu}(\mathrm{n}, \mathrm{f})$ for incident neutron energies up to $20 \mathrm{MeV}$, and predicted PFGS characteristics are compared with values obtained in both recent experiments and model calculations.

\section{Systematics of PFGS characteristics}

According to Ref. [9], the average total $\gamma$-ray energy released in fission $\mathrm{E}_{\gamma, t o t}$ is depending linearly on the prompt fission neutron multiplicity $\bar{v}_{n}$, which is based on the study published in Ref. [11]. There linear dependences were observed between incident neutron energy $E_{n}$ and both prompt fission neutron multiplicity $\bar{v}_{n}$ and the average total $\gamma$-ray energy $\mathrm{E}_{\gamma, \text { tot }}$. From this it is obvious that $\mathrm{E}_{\gamma, \text { tot }}$ has to be proportional to $\bar{v}_{n}$, which "is explained in terms of the linear increase of the average spin of the fragments with their excitation energy" [11]. The latter cited work was then extended from the spontaneous fission of ${ }^{252} \mathrm{Cf}$ to other fissioning systems by including a dependence from both their mass and atomic numbers, $\mathrm{A}$ and $\mathrm{Z}$, respectively [9]. The suggested description for $\mathrm{E}_{\gamma, t o t}\left(\bar{v}_{n}, \mathrm{Z}, \mathrm{A}\right)$ in $\mathrm{MeV}$ is of the form

$$
E_{\gamma, t o t}\left(\bar{v}_{n}, Z, A\right)=\varphi(Z, A) \times \bar{v}_{n}+4.0,
$$

with

$$
\varphi(Z, A)=a_{0}+a_{1} \times Z^{2} A^{1 / 2} .
$$

The parameters $a_{0}$ and $a_{1}$ were determined by a leastsquares fit to available experimental data, while the values for $\bar{v}_{n}$ had been taken from experiments [9]. The average energy per emitted $\gamma$-ray $\epsilon_{\gamma}$ was assumed to be independent from $\bar{v}_{n}$ and depending on $\mathrm{A}$ and $\mathrm{Z}$ according to

a e-mail: andreas .oberstedt@eli-np.ro

$$
\epsilon_{\gamma}(Z, A)=b_{0}+b_{1} \times Z^{1 / 3} A^{-1} .
$$

(c) The Authors, published by EDP Sciences. This is an Open Access article distributed under the terms of the Creative Commons Attribution License 4.0 (http://creativecommons.org/licenses/by/4.0/). 
Here too, the parameters $b_{0}$ and $b_{1}$ were determined by a fit to experimental results. A relation for the average prompt fission $\gamma$-ray multiplicity $\bar{M}_{\gamma}$ may then be inferred by dividing Eq. (1) with Eq. (3) and using Eq. (2). Although different functions might have been used to approximate $\bar{M}_{\gamma}\left(\bar{v}_{n}, \mathrm{Z}, \mathrm{A}\right)$, we have chosen

$$
\bar{M}_{\gamma}\left(\bar{v}_{n}, Z, A\right)=\left(c_{0}+c_{1} \times Z^{5 / 3} A^{-1 / 2}\right) \times \bar{v}_{n},
$$

in order to present experimental values graphically.

Figure 1 gives an overview of all experimental results for (a) $\mathrm{E}_{\gamma, t o t}\left(\bar{v}_{n}, \mathrm{Z}, \mathrm{A}\right),(\mathrm{b}) \epsilon_{\gamma}(\mathrm{Z}, \mathrm{A})$ and (c) $\bar{M}_{\gamma}\left(\bar{v}_{n}, \mathrm{Z}, \mathrm{A}\right)$ in accordance with the above given equations. The dashed (black) lines correspond to the original evaluation [9], based on experimental results that had been reported until 1973 (the corresponding references are also given there), denoted by full drawn (black) circles. The (blue) open squares indicate the results obtained by the Los Alamos/Livermore collaboration [12-15] by using the DANCE detector system [16]. The filled (red) triangles, denoted by "DANCE modified", correspond to the same data as mentioned before. However, as discussed in detail in Ref. [6], the original prompt fission $\gamma$-ray spectra obtained with DANCE lack a considerable amount of $\gamma$-rays with energies below $500 \mathrm{keV}$, most likely due to absorption effects, leading to both lower $\gamma$-ray multiplicities and an overestimation of the average total $\gamma$-ray energy. Since this effect has been quantified, the original values could be corrected, hence modified.

Our results, also recently published [3-6, 10] are shown as (red) full circles for spontaneous and (red) open circles for thermal-neutron induced fission. The good agreement between these experimental values with the modified DANCE values is obvious. The values for $\bar{v}_{n}$ were taken as given in Ref. [9]. Due to obvious discrepancies between the historical and the recently obtained experimental data, a new evaluation seemed to be reasonable on the basis of these new results. It is depicted by solid (red) lines in Fig. 1, obtained by least-squares fits to the data depicted in red, i.e. the open circles and full triangles, weighted with the uncertainties, in case they were given. Obviously, there are differences between our evaluation and the old one by Ref. [9], which of course is reflected by the differences between the recent experimental data and the historical ones. This has to be subject for further theoretical and experimental studies, of which results of the latter then have to be included in the systematics presented here.

For the time being, we draw the conclusion that it should be possible with the revised systematics to both interpolate and extrapolate to any fissioning system in order to estimate their corresponding characteristic properties for thermal-neutron induced and/or spontaneous fission, since the fits were also based on PFGS characteristics measured in exactly the same processes. However, below we emanate from the results of our present evaluation to predict PFGS properties even for fission induced by fast neutrons.

\section{Predictions for fast neutron induced fission}

As concluded above, PFGS characteristics may be estimated for any fissioning system by means of the systematics presented in this work. According to Eqs. (1)
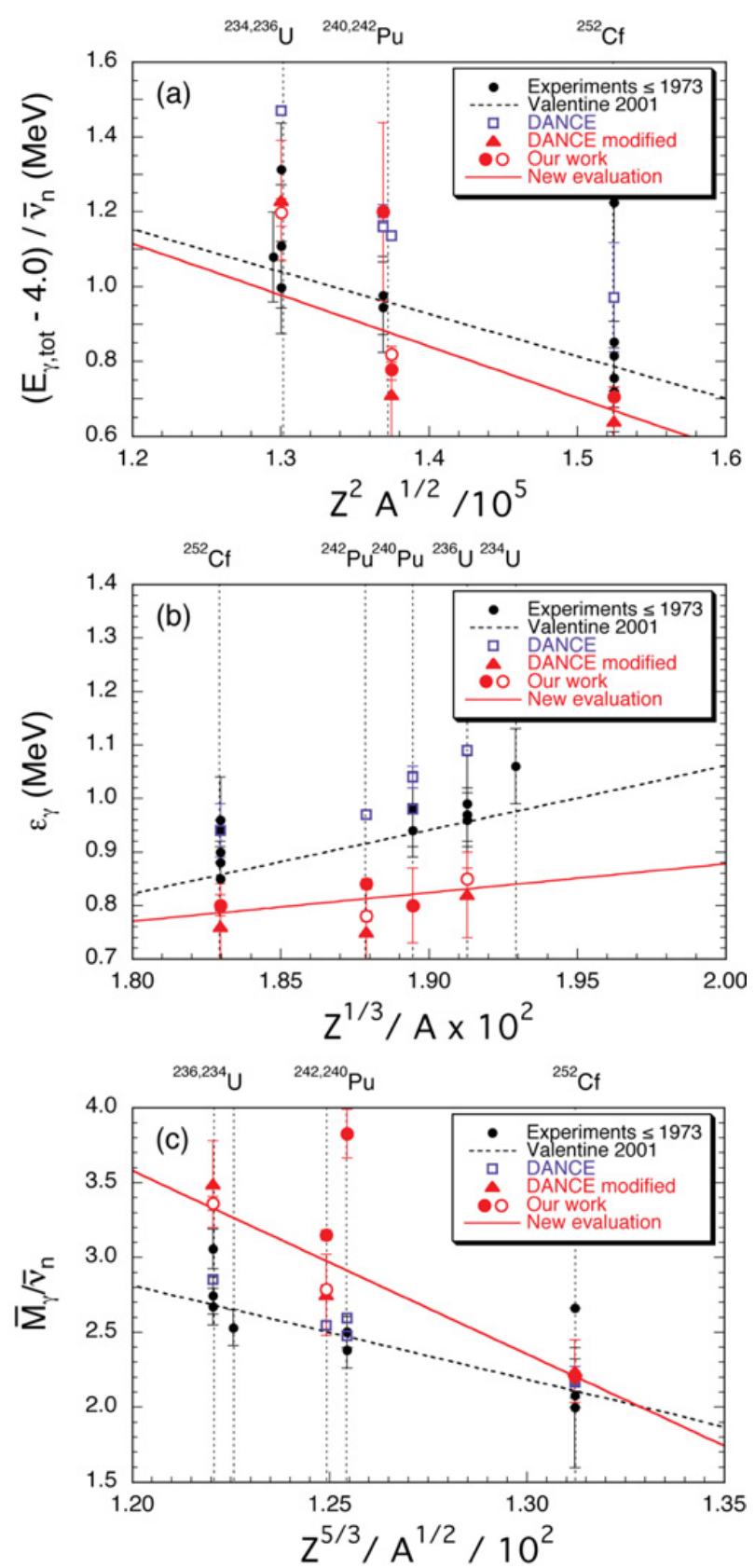

Figure 1. Overview of experimental results for the average total $\gamma$-ray energy released in fission (upper part), the average energy per photon (middle part) and prompt fission $\gamma$-ray multiplicity (lower part) as function of $\mathrm{A}$ and $\mathrm{Z}$ for different fissioning systems. Full (black) circles denote historical results (see Ref. [9] for references), open (blue) squares indicate results obtained with DANCE [12-15], full (red) triangles correspond to modified DANCE data (cf. Ref. [6] for details) and (red) circles represent results from our recent experiments $[3-6,10]$. Here, the full and open circles indicate spontaneous and thermalneutron induced fission, respectively. Also shown are results from evaluations by Ref. [9] (dashed black line) and from this work (solid red line), based on the historical data and recent results, respectively. For the sake of clarity, the corresponding fissioning systems are given, too.

to (4), the only apparent energy dependence is the one that is hidden in the prompt fission neutron multiplicity. Once again, we quote Ref. [11], according to which the linear increase of $\mathrm{E}_{\gamma, t o t}$ with $\bar{v}_{n}$ corresponds to the linear increase of angular momentum with increasing excitation 
energy (cf. Sect. 3). Hence, if the energy dependence of $\bar{v}_{n}$ is known, there is no obvious reason why the validity of this systematics should be restricted to spontaneous or thermal neutron induced fission. The energy dependence of the prompt fission neutron multiplicity is a linear one, according to both Ref. [17] and evaluated data files (e.g. [18]). However, in the considered energy range channels for multi-chance fission may be open, leading to the emission of pre-fission neutrons. Since these neutrons are not emitted from fission fragments but the compound system, they do not contribute to the de-excitation of the fragments in competition with prompt $\gamma$-ray emission. As shown in Ref. [19], they are included in the numbers given in the evaluated files. Hence, pre-fission neutrons have to be assessed and subtracted in order to obtain proper values to be used in the systematics above. As already shown for ${ }^{252} \mathrm{Cf}(\mathrm{sf})$ [11], the total $\gamma$-ray energy released in fission (and the $\gamma$-ray multiplicity) is increasing linearly with the average number of neutrons emitted per fission, i.e. $\bar{v}_{n}$. The same behavior may be inferred for ${ }^{235} \mathrm{U}(\mathrm{n}, \mathrm{f})$ and ${ }^{238} \mathrm{U}(\mathrm{n}, \mathrm{f})$ [17], where a linear increase of both total $\gamma$-ray energy and average prompt neutron multiplicity with incident neutron energy is reported. Still, it was shown for the neutron induced fission of ${ }^{235} \mathrm{U}$ that this is only true as long as the (n, f) channel is considered [19]. Hence, for neutron energies above the neutron separation energy of the compound system, the channels for second, third etc. fission, i.e. (n, nf), (n, 2nf) and so on, may be open and the neutrons emitted prior to fission of the corresponding residual compound systems have to be subtracted from the total number of prompt fission neutrons. Details about the calculations will be found elsewhere [20]; here we have to restrict ourselves to presenting the results of the prediction of PFGS properties.

Figure 2 shows the results as a function of incident neutron energy for $n+{ }^{238} \mathrm{U}$ in terms of (a) average total $\gamma$-ray energy released in fission, (b) average prompt fission $\gamma$-ray multiplicity, and (c) average $\gamma$-ray energy per fission. Our results (solid red lines) are denoted as "Prediction" and shown together with experimental results obtained at CEA DAM/DIF [21] and LICORNE [22]. In addition, results from model calculations with FIFRELIN [23] at $1.8 \mathrm{MeV}$ [24] and Tudora's Point-byPoint model [25-27] as well as a linear fit to an empirical approach from Ref. [17] (dotted black line). In contrast to our results, the latter data do not exhibit any kinks at the thresholds for second and third chance fission (see Fig. 2(a)), which should appear, if the evaporation of neutrons prior to fission had been corrected for. They appear also in the predictions for the average prompt fission $\gamma$-ray multiplicity and the average $\gamma$-ray energy per fission (see Fig. 2(b) and (c), respectively). The (black) dashed line in Fig. 2(c) indicates a constant value according to Ref. [9], where no dependence of $\bar{v}_{n}$ (and, hence, energy) is assumed. There the solid (red) line exhibits kinks and is the result of dividing $\mathrm{E}_{\gamma, t o t}$ with $\bar{M}_{\gamma}$. Which description is to prefer here cannot be decided from the comparison with the experimental values existing so far.

Figures 3 and 4 show the corresponding results of our calculations for the average total $\gamma$-ray energy released in fission for $n+{ }^{235} \mathrm{U}$ and $\mathrm{n}+{ }^{239} \mathrm{Pu}$, respectively. As far as the lighter system is concerned, results from several model calculations are shown too. Values from the
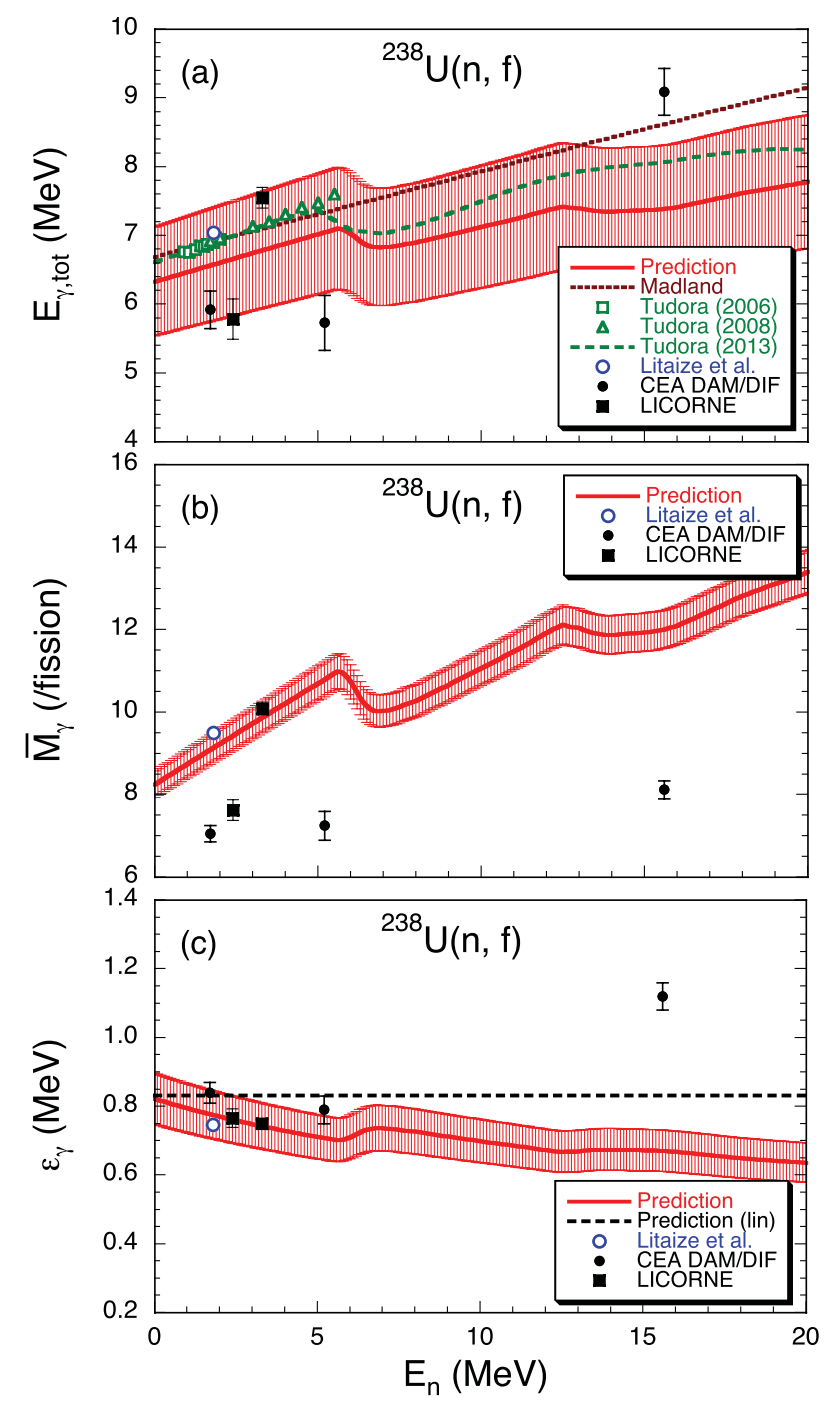

Figure 2. Predicted PFGS characteristics for ${ }^{238} \mathrm{U}(\mathrm{n}, \mathrm{f}$ ): (upper part) average total $\gamma$-ray energy released in fission, (middle part) average prompt fission $\gamma$-ray multiplicity, and (lower part) average $\gamma$-ray energy per fission, all as function of incident neutron energy. The results from this work are depicted as solid (red) lines and compared to experimental results (full black symbols) as well as results from model calculations (denoted by Litaize et al. and Tudora). A linear approximation from Ref. [17] for the average total $\gamma$-ray energy is shown as dotted (brown) line (see text for details).

Point-by-Point model $[29,30]$ are depicted as long dashed and full drawn (green) lines, while the linear empirical approach from Ref. [17] is indicated by a short dashed (brown) line. Again, our predictions agree very well with the cited calculations, apart from the fact that the linear approach does not take into account higher chance fission and, hence, does not exhibit the thresholds for second and third chance fission. The experimental results from both LICORNE [22] and Ref. [31] are also reproduced well. For comparison, several data from thermal-neutron induced fission $[3,13,15,32]$ are shown as well, as full (black) circles, (blue) squares and (orange) diamonds, respectively. The agreement is good, except for the results obtained with DANCE. However, we remind that this deviation was already addressed in Sect. 1. 

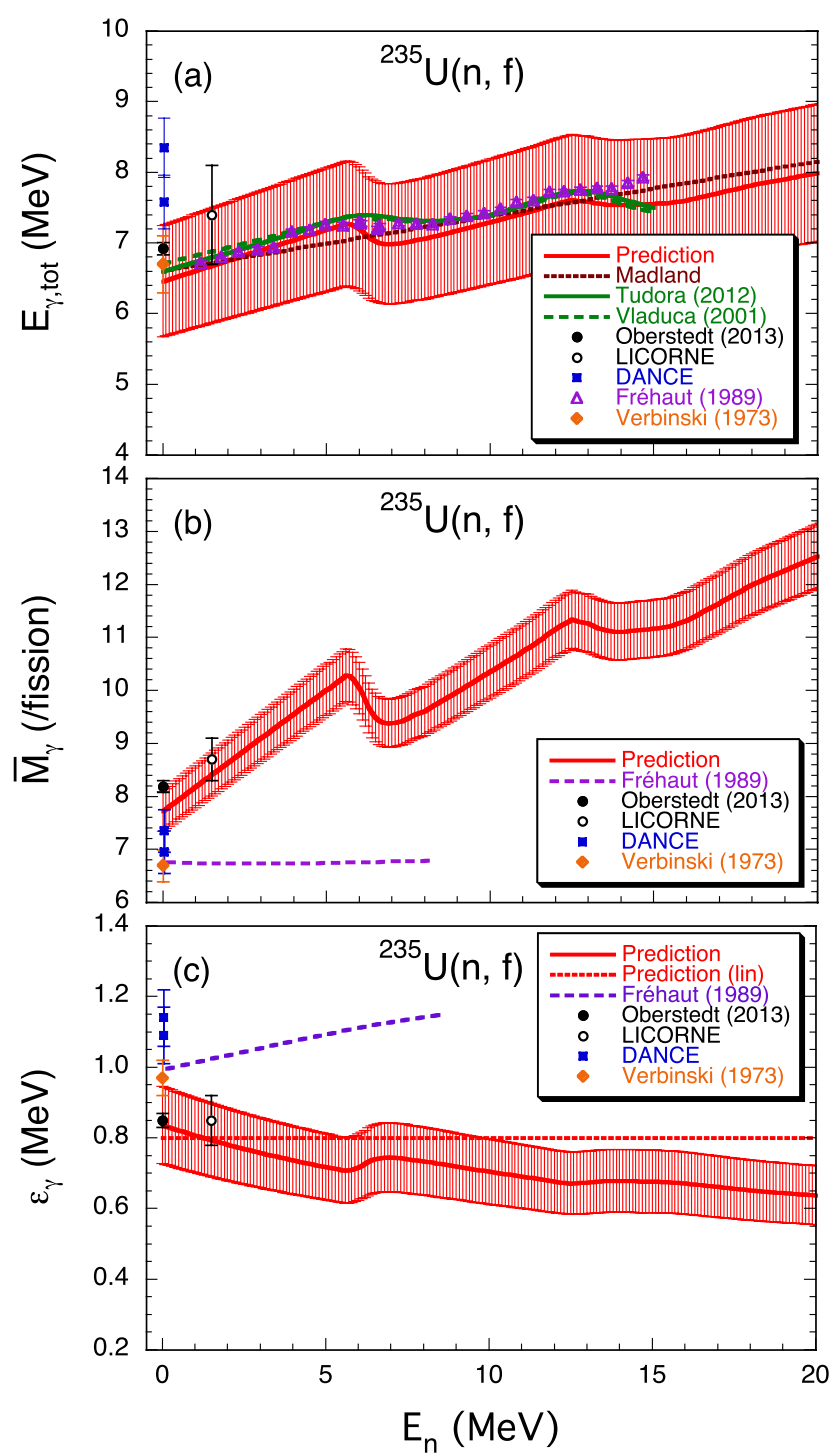

Figure 3. Predicted PFGS characteristics for ${ }^{235} \mathrm{U}(\mathrm{n}, \mathrm{f})$ : (upper part) average total $\gamma$-ray energy released in fission, (middle part) average prompt fission $\gamma$-ray multiplicity, and (lower part) average $\gamma$-ray energy per fission, all as function of incident neutron energy. Again, the results from this work are depicted as solid (red) lines and compared to experimental results (full black symbols) at thermal energies $[3,13,15,32]$, at $1.5 \mathrm{MeV}$ (LICORNE) [22] and up to $15 \mathrm{MeV}$ (Fréhaut). The results from model calculations (denoted by Tudora and Vladuca) $[29,30]$ and a linear approximation (Madland) [17] are shown as well.

In Fig. 4 our results are compared to an evaluation by Ref. [28] and a quadratic fit to the latter [17], with good agreement within the uncertainties. To date experimental data are only available for neutrons of some keV energy and thermal neutrons and depicted by open (blue) squares $[13,15]$, and open and closed (orange) diamonds [32,33], respectively. The agreement is rather good (with the exceptions mentioned above), although the evaluation does not exhibit the same pronounced thresholds for multiple chance fission. However, for all fissioning systems investigated in this work, our calculations lead to consistent results concerning the general behavior of PFGS characteristics as function of incident neutron energy,
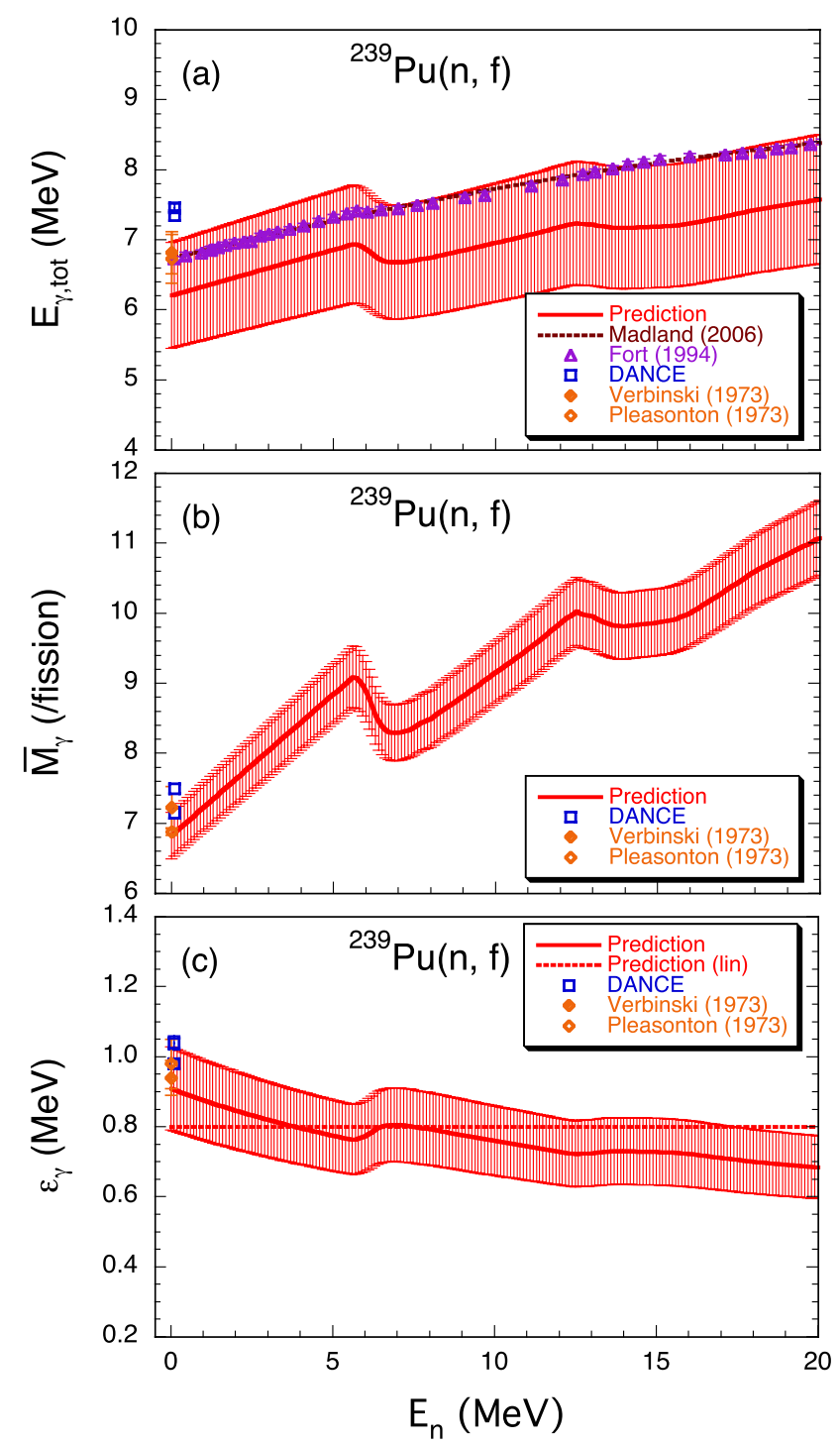

Figure 4. The same as Fig. 3, but for ${ }^{239} \mathrm{Pu}(\mathrm{n}, \mathrm{f})$. Here the few available experimental data at thermal energies from Refs. $[32,33]$ (open and closed orange diamonds) and in the $\mathrm{keV}$ region $[13,15]$ (open blue squares) are complemented by the result of an evaluation [28] and a quadratic fit to the latter [17], depicted as open (purple) triangles and dotted (brown) line, respectively.

which has been confirmed by calculations within the Pointby-Point model, where corresponding results are available.

\section{Conclusions}

Above we have presented predictions for PFGS characteristics from fast-neutron induced fission, based on revised, empirically found systematics as function of both atomic and mass number of the fissioning system. In particular, the systems ${ }^{235} \mathrm{U}(\mathrm{n}, \mathrm{f}),{ }^{238} \mathrm{U}(\mathrm{n}, \mathrm{f})$ and ${ }^{239} \mathrm{Pu}(\mathrm{n}, \mathrm{f})$ have been investigated for incident neutron energies up to $20 \mathrm{MeV}$. Within the certainly still considerable uncertainties, which however should become smaller with more experimental data included in the systematics, one may still observe a rather good agreement between our results and results from both more advanced model calculations, like the ones within the Point-by-Point model, where available 
(see Refs. [26,27]). Calculations with the GEF code [34] will be performed in the near future too. From this we conclude that the new systematics, which was originally found for thermal-neutron induced and spontaneous fission [9] and revised with up-to-date experimental results, may indeed be applied to fission induced by fast neutrons too, as long as the corresponding prompt fission neutron multiplicities are known and correctly used. Of course, experimental data for fast-neutron induced fission is scarce and new measurements are necessary in order test all model calculations. Still, we consider our systematics a useful tool for predicting PFGS characteristics for fissioning systems, which are difficult or even impossible to investigate experimentally.

Part of the results presented in this work was obtained thanks to the financial support by the ERINDA (agreement No. 269499), the EFNUDAT (agreement No. 31027) and the CHANDA programmes (project No. 605203) of the European Commission as well as the COPIL committee of the P2IO for a scientific visit at IPN Orsay. One of the authors (A. O.) also acknowledges the support from the Extreme Light Infrastructure Nuclear Physics (ELI-NP) Phase II, a project co-financed by the Romanian Government and the European Union through the European Regional Development Fund - the Competitiveness Operational Programme (1/07.07.2016, COP, ID 1334), with which this work had been finalized.

\section{References}

[1] Nuclear Data High Priority Request List of the NEA (Req. ID: H.3, H.4), 2006

[2] G. Rimpault et al., Phys. Proc. 31, 3-12 (2012)

[3] A. Oberstedt et al., Phys. Rev. C87, 051602(R) (2013)

[4] S. Oberstedt et al., Phys. Rev. C90, 024618 (2014)

[5] R. Billnert, F.-J. Hambsch, A. Oberstedt, and S. Oberstedt, Phys. Rev. C87, 024601 (2013)

[6] A. Oberstedt, R. Billnert, F.-J. Hambsch, and S. Oberstedt, Phys. Rev. C92, 014618 (2015)

[7] A. Oberstedt, R. Billner, and S. Oberstedt, NEA/NSC/DOC 13, 199-209 (2014)

[8] A. Oberstedt, R. Billnert, and S. Oberstedt, Physics Procedia 59, 24-30 (2014)
[9] T.E. Valentine, Ann. Nucl. Energy 28, 191-201 (2001)

[10] S. Oberstedt et al., Phys. Rev. C93, 054603, 1-6 (2016)

[11] H. Nifenecker et al., Nucl. Phys. A 189, 285-304 (1972)

[12] A. Chyzh et al., Phys. Rev. C85, 021601(R) (2012)

[13] A. Chyzh et al., Phys. Rev. C87, 034620 (2013)

[14] J.L. Ullmann et al., Phys. Rev. C87, 044607 (2013)

[15] A. Chyzh et al., Phys. Rev. C90, 014602 (2014)

[16] M. Heil et al., Nucl. Instr. and Meth. A 459, 229-246 (2001)

[17] D.G. Madland, Nucl. Phys. A 772, 113-137 (2006)

[18] ENDF/B-VII.1 Evaluated Nuclear Data Files, 2011, www.nndc.bnl.gov/exfor/endf02.jsp

[19] Chen Yong-Jing and Liu Ting-Jin, Chin. Phys. C 35(4), 344-348 (2011)

[20] A. Oberstedt, R. Billnert, S. Oberstedt, submitted to Phys. Rev. C

[21] J.-M. Laborie et al., submitted to Phys. Rev. C

[22] M. Lebois and J. Wilson, private communication (2016)

[23] D. Regnier, O. Litaize, O. Serot, Physics Procedia 47, 47-52 (2013)

[24] O. Litaize, O. Serot, D. Regnier, and C. Manailescu, Nuclear Data Sheets 118, 216-219 (2014)

[25] A. Tudora, private communication (2013)

[26] A. Tudora, Ann. Nucl. Energy 53, 507-518 (2013)

[27] A. Tudora, F.-J. Hambsch, S. Oberstedt, Nucl. Phys. A 890-891, 77-101 (2012)

[28] E. Fort, private communications (1994, 2004), see Ref. [17]

[29] G. Vladuca, A. Tudora, Ann. Nucl. Energy 28, 419-435 (2001)

[30] A. Tudora et al., Physics Procedia 31, 43-50 (2012)

[31] J. Fréhaut, IAEA INDC(NDS)-220, 99-111 (1989)

[32] V.V. Verbinski, H. Weber and R.E. Sund, Phys. Rev. C7, 1173-1186 (1973)

[33] F. Pleasonton, Phys. Rev. A213(2), 413-425 (1973)

[34] K.-H. Schmidt, B. Jurado, C. Amouroux, and C. Schmitt, Nuclear Data Sheets 131, 107-221 (2016) 